\title{
Adsorption of binary mixtures of sodium hexadecyl sulfate and ethoxylated octylphenols from aqueous solutions at activated carbon
}

\author{
Victor Maksin, Olga Kochkodan*, Lidiya Kovshun \\ National University of Life and Environmental Sciences of Ukraine, \\ Geroiv Oborony Str. 15, 03041, Kyiv, Ukraine \\ okochkodan@hotmail.com
}

Keywords: adsorption, surfactant, adsorption layer, activated carbon.

Adsorption of binary mixtures of sodium hexadecyl sulfate and oxyethylated octylphenols surfactants from aqueous solutions at activated carbon AG-3 was studied. It is found that the process of mixed adsorption depends on total surfactants concentration in the mixture, the ratio of components in the mixture and their surface activity. Adsorption data is confirmed by measurements of the zeta potential values of activated carbon particles in the surfactants mixtures.

\section{Introduction}

Because of widespread application in numerous colloid-chemical processes such as detergency, flotation, enhanced oil recovery, lubrication, the surface-active substances (surfactants) often enter into the environment, thus adding to the environmental pollution problem, that is of primary global concern. Once in water or soil, the surfactants decompose very slowly over time. These compounds are sorbed on the surface of natural colloidal systems, impeding the processes of biological oxidation and thereby disturb the ecological balance. According to existing international standards, many of the surfactants are assigned to the third and fourth hazard classes [1].

Adsorption is one of the main methods for removal surfactants from water and activated carbon $(\mathrm{AC})$ is most often used in this regard due to its high sorption capacity and low cost [2].

Currently, the adsorption of surfactants at carbon sorbents is the subject of numerous studies, but most of the work in this field is related to adsorption from single-component solutions [3-5]. However, the surfactants mixtures are typically employed for practical applications due to their enhanced performance compared to the solutions, which contain single surfactants only. The main research directions associated with the surfactants mixtures are currently related to development of theoretical approaches to describe colloidal-chemical interactions in the binary surfactants solutions. Most often, the focus is at micelle formation processes in the mixed solutions as well as at adsorption on a solution/air interface [6-8]. As 
for a solid sorbent/solution interface, the studies were conducted mainly with hydrophilic sorbents [9-11].

Among non-polar hydrophobic sorbents, adsorption of nonionic and cationic surfactants mixtures at hydrophobized glass surface of was studied [12, 13]. For AC sorbents, synergistic adsorption of a mixture of anionic and cationic surfactants due to electrochemical attraction of oppositely charged surfactant ions was shown [14].

The aim of this study is to evaluate the main features of adsorption of binary mixtures of ionic and non-ionic surfactants at a porous carbon sorbent such as AC.

\section{Experimental part}

As a sorbent, commercial AC of AG-3 type with total surface area of $6.7 \times 10^{2} \mathrm{~m}^{2} / \mathrm{g}$ evaluated from the adsorption of benzene vapor was used. To remove inorganic impurities, the coal was treated with $2 \mathrm{M} \quad \mathrm{HNO}_{3}$ solutions and concentrated HF accordingly to the procedure described previously [15]. Then the samples were washed with distilled water until no traces of $\mathrm{NO}_{3}{ }^{-}$and $\mathrm{F}^{-}$ions were found in washing water and dried at $105^{\circ} \mathrm{C}$. The total pore volume, the micropores and mezopores volumes evaluated from the adsorption of benzene vapor, were 0.29 , 0.26 and $0.03 \mathrm{~cm}^{3} / \mathrm{g}$, respectively.

Oxyethylated octylphenols such as Triton X45 (TX-45), Triton X-100 (TX-100) and Triton $\mathrm{X}-305$ (TX-305) of the general molecular formula $\mathrm{C}_{8} \mathrm{H}_{17} \mathrm{C}_{6} \mathrm{H}_{4} \mathrm{O}\left(\mathrm{CH}_{2} \mathrm{CH}_{2} \mathrm{O}\right)_{n} \mathrm{H}$ with the degree of oxyethylation $\mathrm{n}=5,9-10$, and 30 , respectively, were used as the non-ionic surfactants. Sodium hexadecyl sulfate (SHS) of the molecular formula $\mathrm{C}_{16} \mathrm{H}_{33} \mathrm{SO}_{4} \mathrm{Na}$ was used as anionic surfactant. The surfactants were purchased from Sigma-Aldrich (USA) and used without further purification.

The critical micelle concentration (CMC) values in the solutions were calculated by using the surface tension isotherms. The surface tension was measured by the Wilhelmy method by balancing a platinum plate by using a torsion balance of BT type at $20{ }^{\circ} \mathrm{C}$. Between the measurements, the plate was pierced in the flame of an alcohol burner. The purity of the plate was controlled by the surface tension of bidistilled water $\left(\sigma=72.5 \mathrm{~mJ} / \mathrm{m}^{2}\right)$. The measurement error was $\pm 0.5 \mathrm{~mJ} / \mathrm{m}^{2}$.

To obtain the adsorption isotherms, the constant sorbent loading was added to the solutions with various initial concentrations of the surfactants and the probes were shaken for specific time. The solutions volume was 0.025 $\mathrm{dm}^{3}$, the sorbent loading was $3 \times 10^{-5} \mathrm{~kg}$. After appropriate adsorption time, that is required for reaching the sorption equilibrium (40 h), the solution was separated from the adsorbent by centrifugation at $4000 \mathrm{rpm}$ for $10 \mathrm{~min}$. The equilibrium concentrations of Triton $\mathrm{X}$ surfactants were determined spectrophotometrically at $\lambda=274 \mathrm{~nm}$, while SHS content in the probes was evaluated by two-phase extraction method with malachite green [16]. 
Malachite green form a complex with SHS, which can be extracted with chloroform followed by measuring of e absorbance of the chloroform phase at $\lambda=616 \mathrm{~nm}[16]$. In our preliminary experiments it was found that each surfactant in the SHS /Triton $\mathrm{X}$ mixtures did not affect the quantification of other component. The measurement error did not exceed $1 \%$ and $5 \%$, respectively.

The specific adsorption value was calculated as follows:

$$
A=\frac{\left(C_{0}-C_{e}\right) \times V}{m}
$$

where $A$ is the adsorption value, $\mathrm{mol} / \mathrm{kg} ; V$ is the volume of surfactant solution, $\mathrm{dm}^{3} ; m$ is the AC loading in the solution, $\mathrm{kg} ; C_{0}$ and $C_{e}$ are the initial and equilibrium concentrations of the surfactant solution, $\mathrm{mol} / \mathrm{dm}^{3}$.

SHS mole fraction in the surfactants mixtures was evaluated from the ratio:

$$
\alpha_{S H S}=\frac{C_{S H S}}{C_{S H S}+C_{T X}}
$$

where, $\alpha_{S H S}$ is the molar fraction of SHS in the mixed surfactant solution, $C_{T X}$ and $C_{S H S}$ are the concentrations of Triton $\mathrm{X}$ and SHS surfactants in the mixed solution, $\mathrm{mol} / \mathrm{dm}^{3}$.

The surfactant solutions with SHS molar fraction $\left(\alpha_{S H S}\right)$ of $0 ; 0.2 ; 0.4 ; 0.6 ; 0.8$ and 1.0 were used.

To evaluate the zeta potential values, the electrophoretic mobility of the sorbent particles ( $\mu$ ) was measured with a Zeta-Meter System $3.0+$ (Zeta Meter Inc., USA) following the procedure as described before [17] and the calculations were performed according to the Smoluchowski equation:

$$
\zeta=\mu \eta / \varepsilon
$$

where $\zeta$ is the zeta potential of the sorbent particles, $\mathrm{mV} ; \eta$ is dynamic viscosity, $\mathrm{Pa} \times \mathrm{s}$, and $\varepsilon$ is dielectric constant. The $\mathrm{pH}$ values of the solutions were within the range of $6.0 \pm 0.5$.

\section{Results and discussion}

The colloid and adsorption characteristics of the used surfactants were determined as described previously $[7,18]$ and are presented in the Table.

Table. Colloid and adsorption characteristics* of the studied surfactants

\begin{tabular}{|c|c|c|c|c|}
\hline Surfactant & $\begin{array}{c}C M C \times \\
10^{4}, \\
\mathrm{~mol} / \mathrm{dm}^{3}\end{array}$ & $\begin{array}{c}\Gamma^{\sigma}{ }_{\text {max }} \\
\times 10^{6}, \\
\mathrm{~mol} / \mathrm{m}^{2}\end{array}$ & $\begin{array}{c}S_{\text {min }}, \\
\mathrm{nm}^{2}\end{array}$ & $\begin{array}{c}-\Delta G^{0}{ }_{\text {mic }} \\
\mathrm{kJ} / \mathrm{mol}\end{array}$ \\
\hline SHS & 9.2 & 2.8 & 0.59 & 17.0 \\
\hline TX-45 & 1.6 & 6.3 & 0.26 & 21.3 \\
\hline TX-100 & 2.4 & 5.5 & 0.30 & 20.3 \\
\hline TX-305 & 3.0 & 4.1 & 0.41 & 19.8 \\
\hline
\end{tabular}

* $C M C$ is critical micelle concentration.

$\Gamma_{\max }^{\sigma}$ is the maximum surface excess at the air/surfactant solution interface.

$S_{\min }$ is the area per the surfactant molecule in the saturated adsorption layer.

$\Delta G^{0}{ }_{m i c}$ is change in the free molar energy of micelle formation

$\Gamma_{\max }^{\sigma}$ was calculated from the Gibbs adsorption equation [19] as :

$$
\Gamma^{\sigma}=-\frac{C}{i R T} \frac{d \sigma}{d C}=-\frac{1}{i R T} \frac{d \sigma}{d \ln C}
$$


where $C$ is surfactant concentration, $\sigma$ is surface tension, $R$ is universal gas constant, $T$ is temperature, $i$ is 1 for a nonionic surfactant, while 2 is for an ionic surfactants, respectively;

$S_{\min }$ was evaluated as [20]:

$$
S_{\min }=\frac{10^{18}}{\Gamma_{\max }^{\sigma} N_{A}}
$$

where $N_{A}$ is the Avogadro number and $\Gamma^{\sigma}{ }_{\max }$ is the maximal adsorption value;

$\Delta G^{0}{ }_{\text {mic }}$ was calculated as [19]:

$$
\Delta \mathrm{G}^{0}{ }_{\text {mic }}=R T \ln C M C
$$

Figure 1 shows the adsorption isotherms of SHS, TX-45, TX-100 and TX-305 surfactants from their individual aqueous solutions at $\mathrm{AC}$ surface as well as changes in zeta potential values of AC particles after adsorption of the surfactants. Adsorption of SHS and Triton X surfactants at $\mathrm{AC}$ is due to hydrophobic interactions between the alkyl radicals of the surfactants and AC surface. Such interactions are recognized as the main driving forces for the adsorption of surfactants at hydrophobic surfaces [9, 20-22].

As can be seen in Fig. 1, the adsorption isotherms in the studied concentration range are of Langmuir type and the curves show the pronounced saturation plateau. Note that with SHS the adsorption saturation was achieved at higher equilibrium concentrations than for Triton $\mathrm{X}$ surfactants (Fig. 1a, b). Also, the maximum adsorption value for SHS $(0.28 \mathrm{~mol} / \mathrm{kg})$ is lesser than appropriate ones for TX-45 $(0.63 \mathrm{~mol} / \mathrm{kg})$ and TX-100 $(0.35 \mathrm{~mol} / \mathrm{kg})$ surfactants, while it is higher compare to TX- $305(0.20 \mathrm{~mol} / \mathrm{kg})$ surfactant.
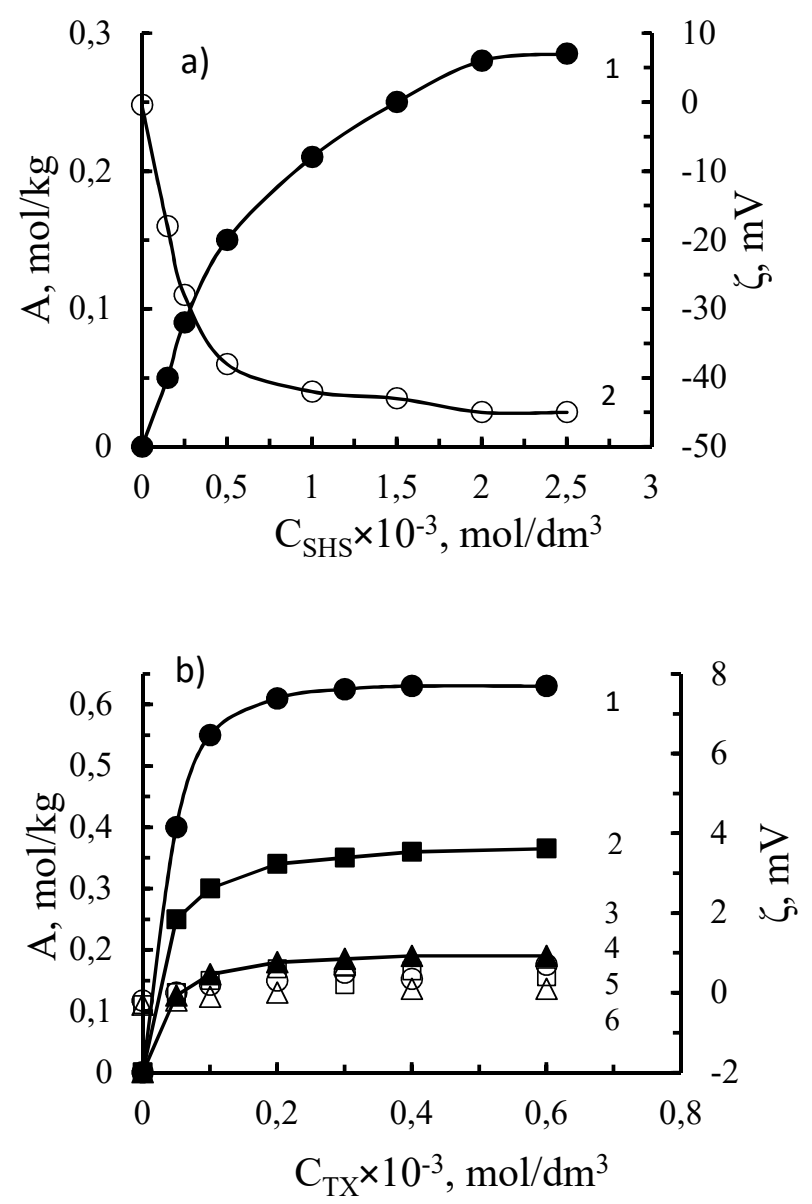

Figure 1. Adsorption isotherms of SHS (a: 1) and Triton X surfactants (b: TX-45 (1), TX-100 (2), TX-305 (3)) from their individual solutions and zeta potential of $\mathrm{AC}$ particles with adsorbed surfactants (a: SHS (2); b: TX-45 (4), TX$100(5)$, TX-305 (6))

Adsorption of non-ionic surfactants depends on the length of the oxyethylated chain of Triton $\mathrm{X}$ molecules, while adsorption of the anionic surfactant is affected by the electrostatic interactions of the similarly charged hydrophilic groups of SHS molecules. Adsorption of SHS molecules notably changes the zeta potential readings of AC particles even at low 
concentrations of the surfactant in the solution (Fig. 1a).

It was found that zeta potential of $\mathrm{AC}$ particles remarkably decreases with an increase in the equilibrium SHS concentration in the solution, reaching a plateau at about $-45 \mathrm{Mv}$ (Fig. 1 a). Obviously, the SHS molecules adsorb at AC surface by their hydrophobic parts while the anionic groups are protruded in the aqueous phase. As a result, the adsorbent particles acquire a negative surface charge.

As seen in Fig. 1b, the zeta potentials values of the sorbent particles in the presence of the nonionic surfactants are very low (within the range of between -0.5 and $+0.7 \mathrm{mV}$ ). These results are similar to the previously published ones [23, 24], where it was shown that the zeta potentials of AC particles in a neutral medium were close to zero. Unlike to SHS, the adsorption of Triton $\mathrm{X}$ surfactants at AC surface practically does not affect zeta potential of AC particles, as Triton $\mathrm{X}$ are non-ionic compounds. Thus, it can be concluded that only SHS adsorption contributes to the zeta potential of AC particles and the change in zeta potential readings can be used to evaluate an increase or decrease in SHS adsorption from its mixtures with the non-ionic surfactants at AC surface.

SHS adsorption from its mixtures with TX45, TX-100, and TX-305 surfactants was studied at different molar fractions of SHS in the mixture: $\alpha_{S H S}=0.2,0.4,0.6$, and 0.8 . Figure 2 indicates, for example, the adsorption isotherm of SHS at AC surface from the binary mixtures with TX-100. SHS adsorption isotherms from SHS/TX-45 and SHS/TX-305 mixtures possess similar features. The results showed that the value of SHS adsorption from the surfactants mixtures depends on the total surfactants concentration in the mixture and the mole fraction of SHS in the mixed solution. At low equilibrium concentrations, SHS adsorption from mixtures is higher compared to SHS adsorption from its single solution. It means that the adsorption of the anionic surfactant increases in the presence of the non-ionic surfactant molecules. This effect is enhanced with an increase in the molar fraction of SHS in the mixture. Such results can be explained by the fact that the incorporation of the molecules of the non-ionic surfactant into the mixed adsorption layer reduces the electrochemical repulsion between the negatively charged SHS ions. This phenomenon leads to increased adsorption of the anionic surfactant from its binary mixtures with the non-ionic surfactants compared to SHS adsorption from its single solution.

On the other hand it was found that SHS presence does not practically affect adsorption of Triton $\mathrm{X}$ surfactants at AC from the binary surfactant mixtures.

With an increase in the total surfactants concentration, SHS adsorption from the mixtures decreases compared to SHS adsorption from its single solution. A similar effect is observed for all studied mixtures of SHS with the non-ionic 
surfactants, but it is the most pronounced for mixtures at $\alpha_{S H S}=0.6$ and 0.8 (Fig. 2).

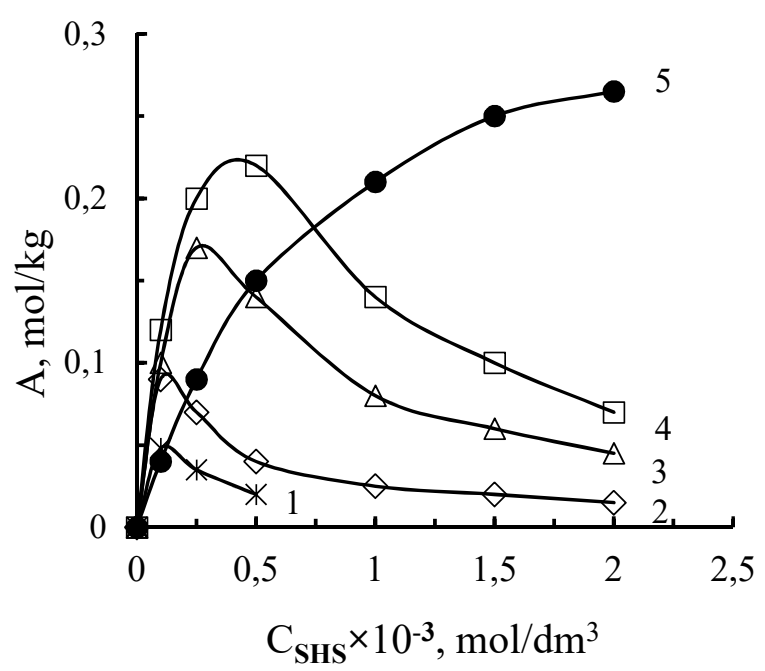

Figure 2. SHS adsorption at AC from mixed SHS/TX-100 solutions at different molar fractions $\alpha_{S H S}$ in the mixture: 0.2 (1), $0.4(2), 0.6(3), 0.8(4)$ and 1.0 (5).

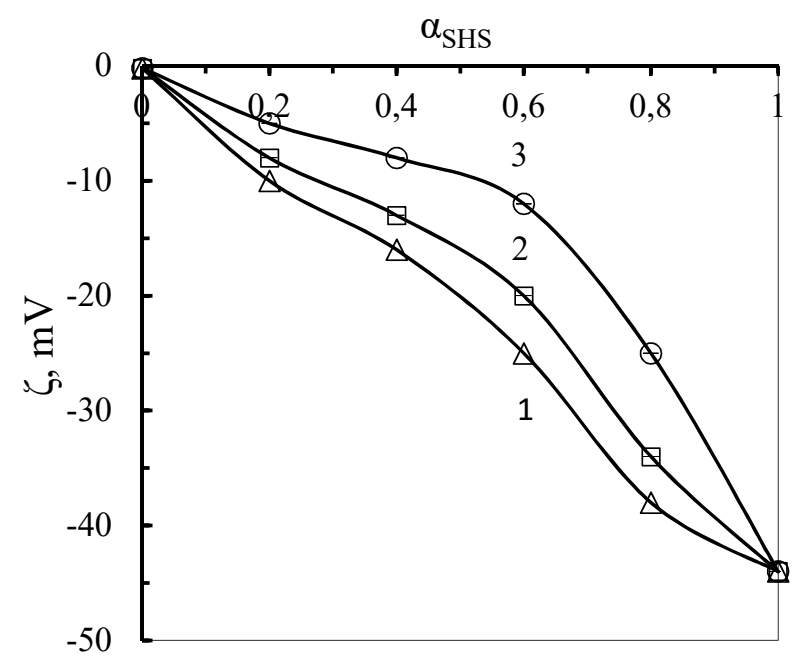

Figure 3. Changes in zeta-potential values of AC particles in SHS mixtures with TX-45 (1), TX-100 (2) and TX-305 (3) versus SHS mole fraction ( $\left.\alpha_{S H S}\right)$ in the mixture.

With an increase in the total surfactants concentration, Triton $\mathrm{X}$ molecules are likely to displace SHS ions from the adsorption layer. Figure 3 shows the change in zeta potential values of $\mathrm{AC}$ particles during SHS adsorption from its mixtures with TX-45, TX-100 and TX305 at the total surfactants concentration of $2.0 \times 10^{-3} \mathrm{~mol} / \mathrm{dm}^{3}$. The zeta potential values of $\mathrm{AC}$ particles increase in an absolute value with an increase in the amount of adsorbed SHS, as shown in Fig. 1. Comparing the zeta potential values at identical molar fractions of SHS in the mixture, it can be concluded about the influence of non-ionic surfactants on SHS adsorption from the surfactants mixtures. The results show that the absolute values of zeta potential and, consequently, SHS adsorption from the binary mixtures with Triton X surfactants increase in the series TX-45>TX-100>TX-305 (Fig.3). Thus, SHS adsorption from the binary mixtures is larger when the surface activity of the non-ionic surfactant, which is present in the mixture, is lower (Table).

\section{Conclusions}

The adsorption of anionic SHS surfactant from its binary mixtures of various compositions with non-ionic Triton X surfactants (TX-45, TX100 and $\mathrm{TX}-305)$ at $\mathrm{AC}$ of $\mathrm{AG}-3$ type was studied. It was found that at low total surfactants concentration, SHS adsorption from the binary mixtures is higher in the presence of non-ionic surfactants than adsorption from the single SHS solution. This can be explained by the fact that the inclusion of the molecules of the non-ionic surfactant in the mixed adsorption layer reduces the electrostatic repulsion between the charged 
hydrophilic groups of SHS molecules and thus promotes its adsorption. With an increase in the total surfactants concentration, the amount of adsorbed SHS from the mixtures is lower compare to SHS adsorption from its single solutions. This is due to partial replacement of SHS ions in the adsorption layer by the molecules of the non-ionic surfactant. The results of measuring the zeta potential values of $\mathrm{AC}$ particles in the surfactant mixtures showed that SHS adsorption from the binary mixtures increases with decreasing the surface activity of the non-ionic surfactant.

\section{Acknowledgements}

The authors wishes to acknowledge the support of Ministry of Education and Science of Ukraine under Grant No. 110/103-F

\section{References}

[1] Ying GG. Fate, behavior and effects of surfactants and their degradation products in the environment. Env. Intern.

2006;32(3):417-431.

doi:10.1016/j.envint.2005.07.004

[2] Sweetman M, May S, Mebberson N, Pendleton P, Vasilev K, Plush S, Hayball J. Activated Carbon, Carbon Nanotubes and Graphene: Materials and Composites for Advanced Water Purification. J Carbon Res. 2017;3(4):1842. doi:10.3390/c3020018

[3]. Krivova M, Grinshpana D, Hedin N. Adsorption of CnTABr surfactants on activated carbons. Colloids and Surfaces A: Physicochem Eng Aspects. 2013;436:62-70. doi:10.1016/j.colsurfa.2013.05.063

[4] Lyu X, You X, He M, Zhang W, Wei H, Lia L, Heb Q. Adsorption and molecular dynamics simulations of nonionic surfactant on the low rank coal surface. Fuel 2018; 211:529-534. doi: 10.1016/j.fuel.2017.09.091

[5] Marsalek R, Pospisil J, Taraba B. The influence of temperature on the adsorption of CTAB on coals. Colloids and Surfaces A: Physicochem Eng Aspects. 2011;383:8085. doi: 10.1016/j.colsurfa.2011.01.012

[6] Szymczyk K, Zdziennicka A, Krawczyk J, Janczuk B. Mutual influence of cetyltrimethylammonium bromide and Triton X-100 on their adsorption at the water-air interface. J Chem Termodyn. 2013;59:35-42. doi:10.1016/j.jct.2012.12.004

[7] Kochkodan OD, Antraptseva NM, Maksin VI. Micelle formation and adsorption in aqueous solutions of binary mixtures Triton X-100 with dodecylpyridinium bromide and sodium dodecylsulfate. Ukr Khim J. 2018; 84(9):40-46. [in Ukrainian]

[8] Ivanova NI. Micelloformation and surface properties of aqueous solutions of binary mixtures of Twin 80 and cetyltrimethylammonium bromide. Vestn. Moscow Univ. Chem. 2012;53(1):44-49. [in Russian]

[9] Zhang R, Somasundaran P. Advances in adsorption of surfactants and their mixtures at solid/solution interfaces. Adv Colloid Interface Sci. 2006;123-126:213229. doi: 10.1016/j.cis.2006.07.004

[10] Woods DA, Petkov J, Bain CD. Surfactant Adsorption Kinetics by Total Internal Reflection Raman Spectroscopy. 2. CTAB and Triton X-100 Mixtures on Silica. J Phys Chem. B. 2011;115:7353-7363. doi:10.1021/jp201340j

[11] Chang Z, Chen X, Peng Y. The adsorption behavior of surfactants on mineral surfaces in the presence of electrolytes - A critical review. Minerals Eng. 2018;121:66-76. doi: 10.1016/j.mineng.2018.03.002

[12] Kostenko AV, Soboleva OA. Adsorption modification of a hydrophobic solid surface with solutions of surfactant mixtures. Vestn. Moscow Univ. Chem. 2008;49 (5): 313-318. [in Russian]

[13] Ivanova NI, Parfenova AM, Amelina EA. The interaction of non-polar surfaces in solutions of mixtures 
of cationic and nonionic surfactants. Vestn. Moscow Univ. Chem. 2007;48(3):182-187. [in Russian]

[14] Xiao JX, Zhang Y, Wang C, Zhang J, Wang CM, Bao YX, Zhao ZG. Adsorption of cationic-anionic surfactant mixtures on activated carbon. Carbon 2005;43:1032-1038. doi: 10.1016/j.carbon.2004.11.039

[15] Koganovskiy AM, Levchenko TM, Kirichenko VA. Adsorption of soluble compounds. Kiev: Naukova dumka; 1977. [in Russian]

[16] Sun J, Du K, Fu L, Gao J, Zhang H, Feng W, Ji P. Sodium Hexadecyl Sulfate as an Interfacial Substance Adjusting the Adsorption of a Protein on Carbon Nanotubes. ACS Appl. Mat. Interf. 2014;6:15132-15139. doi.org/10.1021/am5032715

[17] Sis H, Birinci M. Effect of nonionic and ionic surfactants on zeta potential and dispersion, properties of carbon black powders. Coll. Surf. A: Phys. Eng. Aspects. 2009; 341: 60-67. doi:10.1016/j.colsurfa.2009.03.039

[18] Kochkodan OD, Maksin VI, Antraptseva NM, Semenenko TS. Effect of $\mathrm{pH}$ and ionic strength of solution on the colloidal-chemical properties of binary mixtures of surfactnts. Biores. i pryrodocoryst. [Bioresources and nature management]. 2018;10(5-6):113-121. [in Ukrainian]

[19] Rosen MJ, Kunjappu JM. Surfactants and interfacial phenomena. New Jersey: Jon Willey and Sons Inc.; 2012.

[20] Parfitt GD, Rochester CH. Adsorption from Solution at the Solid/Liquid Interface. London: Academic press; 1983.

[21] Klimenko NA, Karmasina TV, Kochkodan OD. Alteration of degree of water structuring and its influence on processes of micellization and adsorption of surfactants. Ukr Khim J. 2001;67(1): 82-87. [in Russian]

[22] Tiberg F, Brinck J, Grant L. Adsorption and surface-induced self-assemblyof surfactants at the solid aqueous interface. Cur Opin Coloid Inter Sci. 2000;4:411419. doi: 10.1016/S0043-1354(98)00109-2

[23] Julien F, Baudu M, Mazet M. Relationship between chemical and physical surface properties of activated carbon. Water Res. 1998;32(11):3414-3424. doi:10.1016/S0043-1354(98)00109-2

[24] Kitahara A, Wamanabe A. Electrical phenomena at interfaces: Fundamentals, Measurements, and Applications. New York: Marcel Dekker Inc.; 1984. 